\title{
Information Retention by Stringy Black Holes
}

\author{
John Ellis, Nick E. Mavromatos* \\ Theoretical Particle Physics and Cosmology Group, Department of Physics, \\ King's College London, Strand, London WC2R 2LS, U.K; \\ Theory Division, Physics Department, CERN, CH 1211 Geneva 23, Switzerland \\ E-mail: John.Ellis@cern.ch, nikolaos.mavromatos@kcl.ac.uk

\section{V. Nanopoulos ${ }^{\dagger}$}

George P. and Cynthia W. Mitchell Institute for Fundamental Physics and Astronomy, Texas A\&M University, College Station, TX 77843, USA;

Astroparticle Physics Group, Houston Advanced Research Center (HARC),

Mitchell Campus, Woodlands, TX 77381, USA;

Academy of Athens, Division of Natural Sciences, Athens 10679, Greece

E-mail: dimitri@physics.tamu.edu

KCL-PH-TH/2015-51, LCTS/2015-38, CERN-PH-TH/2015-258, ACT-09-15, MI-TH-1539

\begin{abstract}
Building upon our previous work on two-dimensional stringy black holes and its extension to spherically-symmetric four-dimensional stringy black holes, we show how the latter retain information. A key rôle is played by an infinite-dimensional $W_{\infty}$ symmetry that preserves the area of an isolated black-hole horizon and hence its entropy. The exactly-marginal conformal worldsheet operator representing a massless stringy particle interacting with the black hole necessarily includes a contribution from $W_{\infty}$ generators in its vertex function. This admixture manifests the transfer of information between the string black hole and external particles. We discuss different manifestations of $W_{\infty}$ symmetry in black-hole physics and the connections between them.
\end{abstract}

18th International Conference From the Planck Scale to the Electroweak Scale 25-29 May 2015

Ioannina, Greece

${ }^{*}$ Work supported in part by the European Research Council via the Advanced Investigator Grant 267352 and by the UK STFC via the Research Grant ST/L000326/1.

${ }^{\dagger}$ Work supported in part by the DOE Research Grant DE-FG02-13ER42020. 


\section{Introduction}

The black-hole information problem requires no introduction. Decades ago, Bekenstein [1] and Hawking [2] discovered that four-dimensional black holes have thermodynamical properties such as temperature and non-zero entropy corresponding to a mixed quantum-mechanical state. Hawking, in particular, then argued [3] that information would be lost across the black-hole horizon, giving rise to a transition from a pure to a mixed state.

The advent of string theory, and in particular Witten's construction [4] of a two-dimensional black hole solution using an $\mathrm{SU}(1,1) / \mathrm{U}(1)$ coset structure [5, 6], coupled with dualities [7] and followed by the construction of four-dimensional stringy black holes using D-branes [8, 9], provided opportunities to probe the black-hole information problem in an explicit theoretical laboratory. We have argued [10] that two-dimensional black holes carry an infinite set of 'hairy' $W$ quantum numbers that preserve in principle the lost information, though in practice this information is inaccessible. We have also argued that these observations can be extended to spherically-symmetric four-dimensional black holes [12], whose horizon geometry is encoded in a similar $\mathrm{SU}(1,1) / \mathrm{U}(1)$ coset structure, accompanied by a similar infinite-dimensional $W$ symmetry and an associated infinite set of 'hair' that is measurable in principle [13].

Constructions using D-branes provided explicit examples of four-dimensional black-hole solutions whose microstates could be counted [8], giving numbers consistent with the BekensteinHawking entropy and suggesting that indeed the 'lost' information could in principle be retained. However, there still remained the issues how the information was transferred to and from these microstates, in what form it was stored, and whether the information transferred into the microstates could in practice be extracted, or whether it was in reality lost.

An interesting approach to addressing these issues has recently been taken in a series of papers by Strominger and collaborators $[14,15]$. They have shown that spherically-symmetric fourdimensional black holes carry an infinite set of gravitational 'hair' associated with BBMS supertranslations $[16,17]$ on the retarded null infinity $\mathscr{I}^{+}$, corresponding to vacua that differ by the addition of soft gravitons and could be measured via the gravitational memory effect. They also found an infinite set of inequivalent electromagnetic gauge configurations corresponding to electromagnetic hair and differing by the addition of soft photons. It has recently been suggested by Hawking [18] that the apparent information loss paradox might be resolved by considering supertranslations on the horizon, with the information 'lost' by incoming particles being recoverable in principle, though lost in practice.

This proposal raises many questions, including whether supertranslations (together with superrotations and the corresponding electromagnetic gauge configurations) are capable of encoding all the information carried by the incoming particles [19], as well as the relation to the stringy description of black holes, the details of the mechanism for information transfer to and from the black hole, and whether information is really lost. We mention here for completeness the recent work of 't Hooft [20], where it is argued that the black-hole horizon should be viewed as an apparent world-sheet of an induced string theory. This is to be contrasted with the spirit of our approach in this paper, where we start from string theory at a fundamental level. We also mention the recent work of Polchinski [21] in which a shock-wave approximation was used to calculate the shift on a generator of the horizon caused by an ingoing wave packet, which is similar in spirit to our analysis 
below of the supertranslation of the horizon (viewed as a recoiling D-brane) induced by infalling matter. Other works in similar spirit include [22, 23], where the back-reaction of matter falling onto the black hole horizon (and fluctuations of the latter) is argued to play an important rôle in retaining information.

In this paper we review our previous arguments about the importance of $W_{\infty}$ symmetry [24, 25] ${ }^{1}$ for 'balancing the information books'. In particular, we recall that a massless stringy particle interacting with the black hole is represented by a conformal operator on the world-sheet of the string, which is exactly marginal only if a contribution from $W_{\infty}$ generators is included in its vertex function $[10,6]$. Without this contribution, the corresponding renormalization-group (RG) $\beta$ function is non-zero, leading to an inexorable increase in entropy. As we recall $[10,11], w_{\infty}$ (which is the classical limit of the quantum $W_{\infty}$ symmetry) is the algebra of transformations that preserve the two-dimensional phase-space volume of massless ('tachyonic') stringy matter propagating in the background of a stringy black hole.

Moreover, these quantum $W$-algebras are symmetries of the quantum scattering matrix of the corresponding two-dimensional string theory $[26,6]$, in the sense that the operator product expansion between two appropriate vertex operators reproduce the corresponding $W$-algebra. In the flat space-time case (in which case the string theory is just a two-dimensional Liouville theory) the operators corresponding to the discrete higher-spin operators of the $W$ algebra are discretized 'tachyon' operators. However, as already mentioned, in the presence of a black hole, at the quantum level, the corresponding $W_{\infty}$ symmetries necessarily mix massless and massive (topological, delocalised) stringy states [6]. The admixture of $W_{\infty}$ generators in the exactly marginal vertex operator of a massless string excitation shows how information is transferred between a stringy black hole and external particles.

We discuss below the embedding of the two-dimensional coset describing the singularity in a four-dimensional space-time [12] with the structure $\mathrm{SU}(1,1) / \mathrm{U}(1) \otimes S^{2}$, where $S^{2}$ is a twodimensional manifold with the topology of the sphere that is to be identified with the horizon of the four-dimensional black hole. Under certain circumstances specified below, in addition to the quantum $W$-symmetries that leave the (quantum-gravity) scattering matrix invariant, and are associated with the discrete (topological) states of the two-dimensional coset substructure, there is also a classical $w_{\infty}$ algebra of symmetry transformations of the horizon coordinates that preserves the area of the horizon of an isolated spherically-symmetric four-dimensional black hole, so that its entropy (which is known to be a Noether charge [27]) is conserved. The precise relation between these two different $W$-symmetries is not yet completely understood. It becomes evident, however, in the case where the horizon of the black hole is represented as a thick (recoiling) brane, which is known to correspond to an $\mathrm{SU}(\infty)$ gauge theory $[28,29]$. The black hole emerging in the low-energy limit of this string/brane theory is the infinitely-coloured $\mathrm{SU}(\infty)$ black hole discussed in [30], which is reviewed below. In this case, the gauge states on the horizon can be represented as open-string states whose ends are attached to the horizon brane, which then carry the $\mathrm{SU}(\infty)$ charges. It is known that classically such $\mathrm{SU}(\infty)$ symmetries are isomporphic to the $w_{\infty}$ algebra that preserves a two-dimensional area, which can in this case can be identified with the horizon

\footnotetext{
${ }^{1}$ This becomes $W_{1+\infty}$, if one includes conformal spin-one states. We denote by lower-case $w_{\infty}$ the classical symmetry, and by upper-case $W_{1+\infty}$ its quantum counterpart, which includes an additional conformal spin-one state.
} 
area of the spherically-symmetric, infinitely-coloured black hole.

\section{Entropy Increase and Non-Critical String}

In the usual treatment of critical string theory, entropy remains constant and, in particular, pure initial states remain pure during the time evolution. A stringy discussion of the possibility of information loss therefore requires going beyond the critical string framework. Accordingly, we now recall our arguments [11] on entropy increase in non-critical string theory, i.e., in a string model where conformal invariance on the world-sheet surface $\Sigma$ is broken by relevant operators.

In such a case, the world-sheet dynamics depends on interaction terms that represent noncritical deformations of the form

$$
S_{\sigma}=S_{\sigma}^{\star}+\int_{\Sigma} d^{2} \xi \sqrt{-\gamma} g^{i} V_{i}
$$

where $S_{\sigma}^{\star}$ is a conformal fixed-point $\sigma$-model action, summation over repeated indices is implied, $\gamma$ is a world-sheet metric, and the set of $\left\{g^{i}\right\}$ is an (infinite in general) set of target-space fields associated with the corresponding vertex operators $V_{i}$. These target-space fields include the lowestlying (massless) string modes, i.e., the graviton, dilaton and antisymmetric tensor fields, the scalar 'tachyons' (in the case of a non-supersymmetric target space), as well as the infinity of higher-spin states ${ }^{2}$.

We introduce a global world-sheet RG scale $\mu$, and define $\mathscr{T} \equiv \ln \mu$. We consider a density matrix $\rho\left(g^{i}\right)$ describing the propagation of a string representing a matter state in the deformed target-space background corresponding to the world-sheet action (2.1). This density matrix is a generic function of the background fields $\left\{g^{i}\right\}$. The renormalizability of the world-sheet twodimensional theory implies that any explicit dependence of $\rho\left(g^{i}\right)$ on $\mathscr{T}$, represented by the corresponding partial derivatives, is compensated by the 'running' of the renormalised $g^{i}$ with $\mathscr{T}$, leading to the following world-sheet RG equation:

$$
0=\frac{d}{d \mathscr{T}} \rho\left(g^{i}\right)=\frac{\partial}{\partial \mathscr{T}} \rho\left(g^{i}\right)+\frac{d g^{j}}{d \mathscr{T}} \frac{\partial \rho}{\partial g^{j}} .
$$

It should be understood that the $\partial_{i} \equiv \frac{\partial}{\partial g^{i}}$ denote functional derivatives $\frac{\delta}{\delta g^{i}}$ with respect to the corresponding fields $g^{i}$ in target space-time. Roughly speaking,

$$
\frac{d g^{j}}{d \mathscr{T}} \equiv \beta^{i}
$$

is a RG $\beta$ function for the 'coupling' $g^{i}$ of the two-dimensional world-sheet field theory ${ }^{3}$.

\footnotetext{
${ }^{2}$ In a two target-space-dimensional setting, the only propagating multiplet consists of massless scalar fields (misleadingly called 'tachyons'), whereas the graviton and higher-spin multiplets are topological states with discrete momenta. As we discuss later, such topological states exist also in higher-dimensional target space-times, so their presence is rather generic.

${ }^{3}$ More strictly speaking, in string theory the target-space dependences of the 'couplings' $g^{i}$ imply some diffeomorphism variations, which lead to the replacement of the corresponding $\beta^{i}$ RG functions by the corresponding Weyl anomaly coefficients, but such complications are not relevant for our main arguments below, so we omit them here. For details see [10].
} 
Representing (in a heuristic way) the density matrix by its Gibbs (equilibrium) form in target space, one has, in terms of the effective target-space string Hamiltonian

$$
\rho\left(g^{i}\right)=\operatorname{Tr} e^{-\beta H},
$$

where $\beta$ is an effective 'temperature' in target space, e.g., the Hawking temperature in the case of a black hole. We assume that the Tr operation commutes with $\partial / \partial \mathscr{T}$, which is consistent with the interpretation of the RG scale $\mathscr{T}$ as time (see below). We then deduce that the von Neumann (entanglement) entropy,

$$
\mathscr{S} \equiv-\operatorname{Tr}(\rho \ln \rho)
$$

varies with $\mathscr{T}$ as

$$
\frac{\partial \mathscr{S}}{\partial \mathscr{T}}=-\operatorname{Tr}\left[\frac{\partial \rho}{\partial \mathscr{T}} \ln \rho\right]-\operatorname{Tr}\left[\frac{\partial \rho}{\partial \mathscr{T}}\right] .
$$

In [11] we have used Liouville dressing to restore conformal invariance on the world sheet, and argued that the Liouville mode may be regarded as a local (on the world-sheet) covariant RG scale, $\rho(\sigma)$, where $\sigma$ denotes the world-sheet coordinates.

In the presence of such a local world-sheet RG scale, there are counterterms in the $\sigma$-model action of the form [11]:

$$
\int_{\Sigma} \partial_{\alpha} g^{i} \mathscr{G}_{i j} \partial_{\alpha} g^{j}
$$

where $\alpha=1,2$ span the world-sheet coordinates. The only dependences on them of the 'renormalised' couplings $g^{i}$ occur through their dependences on the local RG scale $\rho(\sigma)$, so we can write (2.7) as

$$
\int_{\Sigma} \partial_{\alpha} \rho \partial_{\alpha} \rho \hat{\beta}^{i} \mathscr{G}_{i j} \hat{\beta}^{j}
$$

where $\hat{\beta}^{i} \equiv d g^{i} / d \rho$ is a Weyl-anomaly coefficient. i.e., a world-sheet RG $\beta$-function with respect to the local RG scale. The quantity $\mathscr{G}^{i j}$ in $(2.7,2.8)$ acts as a Zamolodchikov 'metric' in the theory space $\left\{g^{j}\right\}$ of the $\sigma$-model, i.e., in the space of target-space background fields. From conformalfield theory considerations [11] that we do not discuss here, one has

$$
\frac{d}{d \rho} Q^{2}[g] \propto \hat{\beta}^{i} \mathscr{G}_{i j} \hat{\beta}^{j}
$$

where $Q^{2}=C[g]-c^{\star}$ is the central-charge deficit of the corresponding non-critical string theory with central charge $C[g]$ that is a functional of $\left\{g^{i}\right\}$, and the quantity $c^{\star}$ is the central charge at a conformal point.

The local RG scale $\rho$ plays the rôle of a Liouville mode, which dresses the renormalised couplings $g^{i}$ in such a way so as to restore criticality in the $D+1$ dimensional target space: we find from (2.8) that the scale $\rho$ is a propagating $\sigma$-model scalar field, and thus its zero mode on the world-sheet may be interpreted as an extra target-space-time coordinate. The sign of $Q^{2}$ depends whether the theory is subcritical or supercritical, with the supercritical case corresponding to $Q^{2}>0$. Actually, as discussed in detail in [11], the dependence of $Q^{2}[g]$ on the local RG scale $\rho$ is such that the derivative with respect to the world-sheet zero mode of $\rho, \rho_{0}$, which is also identified with the global RG scale $\mathscr{T}=\rho_{0}$, obeys $d Q^{2} / d \rho_{0} \propto-Q^{2}+\ldots$, as a result of the fact that $Q^{2}$ is proportional to the $\sigma$-model partition function, in which the RG scale couples to the 
world-sheet curvature as $\int D \rho e^{-\int_{\Sigma} \rho R^{(2)}}$, where $\int_{\Sigma} R^{(2)}$ is the Euler characteristic of the world-sheet manifold. In this way, one recovers from $(2.8,2.9)$ via perturbation theory the kinetic term of the Liouville action, i.e., a term in the $\sigma$-model-field-theory action $S_{\sigma}$ of the form

$$
S_{\sigma} \ni-\int_{\Sigma} \partial_{\alpha} \rho \partial_{\alpha} \rho\left(Q^{2} \hat{+} \ldots\right)
$$

where, in our convention [11], the corresponding kinetic terms of the spatial target coordinates of the string $X^{I}, I=1, \ldots 3$ have a minus sign.

In this case the zero mode of the world-sheet RG flow of this super-critical string can be identified (up to a sign) with the temporal flow in the target space-time [11]:

$$
t=-\mathscr{T}\left(=-\rho_{0}\right)
$$

With this identification, the second term on the right-hand side of (2.6) would vanish in a theory with energy conservation on the average, as for quantum black holes.

Then, using (2.2), (2.3) and (2.11), we obtain from (2.6):

$$
\frac{\partial \mathscr{S}}{\partial t}=-\operatorname{Tr}\left[\frac{\partial \rho}{\partial t} \ln \rho\right]=\operatorname{Tr}\left[\beta \frac{\partial H}{\partial t} \rho \ln \rho\right]=-\operatorname{Tr}\left[\beta \beta^{i} \frac{\partial H}{\partial g^{i}} \rho \ln \rho\right],
$$

where we have used (2.4) and the fact that $H\left(g^{i}\right)$ is a functional of the background fields.

In a string theory setting, the effective Hamiltonian $H$ may be identified (up to a sign) with the effective action $\Gamma$, and it is known in this case that its field variations are proportional off-shell to the $\beta^{i}$ (which is equivalent to the well-known statement that the string conformal invariance conditions on the world-sheet are equivalent on-shell to the equations of motion of the target-space effective action):

$$
\frac{\partial H}{\partial g^{i}}=-\frac{\partial \Gamma}{\partial g^{i}}=-\mathscr{G}_{i j} \beta^{j}
$$

where $\mathscr{G}_{i j}$ is the Zamolodchikov metric in the space of string models [11]. From (2.12), (2.13) we then obtain

$$
\frac{\partial \mathscr{S}}{\partial t}=\beta \beta^{i} \mathscr{G}_{i j} \beta^{j} \mathscr{S} .
$$

Within the context of a unitary world-sheet $\sigma$-model, corresponding to a Euclidean target spacetime as can be used to represent a finite-temperature black holes, the factor $\beta^{i} \mathscr{G}_{i j} \beta^{j}>0$. In this case, then, (2.14) implies a monotonic entropy increase of the (positive) entropy $\mathscr{S}>0$ as time increases, i.e., during the evolution from infrared to ultraviolet on the world sheet, whenever a string propagates in a non-conformal background.

We use this result in the following, in the specific context of strings propagating in target-space black hole backgrounds.

\section{3. $W_{\infty}$ Symmetry Retains Information in a Two-Dimensional Stringy Black Hole}

The prototypical stringy black hole solution in two dimensions [4] is characterized by a worldsheet Wess-Zumino-Witten (WZW) $\sigma$-model formulated on the coset space $S L(2, R)_{k} / U(1)$, where 
$k=9 / 4$ is the Kac-Moody algebra level. The conformal invariance condition for this world-sheet $\sigma$-model induces a target-space metric corresponding to a Euclidean black hole background

$$
d s^{2}=d r^{2}+\tanh ^{2} r d \tilde{\theta}^{2},
$$

where $(r, \theta)$ are two-dimensional coordinates, $r$ being the radial coordinate and $\tilde{\theta}$ a compact 'angular' coordinate that plays the rôle of an external temperature variable: it should not be confused with a four-dimensional angular variable. The space time (3.1) looks like a semi-infinite cigar, and may be elevated to four dimensions via a similar formula with additional angular variables, as described in Section 4.

The spectrum of stringy excitations in the two-dimensional stringy black hole includes background massive topological states that possess a quantum $W_{1+\infty}$ symmetry $[24,6]{ }^{4}$. This leads to an infinity of conserved charges ('hair') for the black hole system, rendering it completely integrable [4]. The $W_{1+\infty}$ symmetry corresponds classically to a classical infinite-dimensional $w_{\infty}$ algebra of diffeomorphisms that preserve a two-dimensional area form, which could correspond to the surface of a sphere $S^{2}$ or some other two-dimensional manifold. In the case of the twodimensional stringy black hole examined in this Section, this is a symplectic phase space 'area' form, which corresponds to the Hamiltonian of a particle system in the near-horizon geometry of the black hole. As we discuss later, this algebra appears also in the case of four-dimensional stringy/brany solutions interpolating between black holes and AdS spaces.

Generically, a symplectic area two-form $\Omega$ corresponding to coordinates $x, y$ :

$$
\Omega=d y \wedge d x
$$

is invariant under classical symmetry transformations that leave it invariant. These area-preserving diffeomorphisms are generated by the quantities

$$
v_{m}^{\ell}=y^{\ell+1} x^{\ell+m+1}
$$

where $\ell$ and $m$ are integers. The Poisson brackets of these generators satisfy the classical $w_{\infty}$ algebra

$$
\left\{v_{m}^{\ell}, v_{m^{\prime}}^{\ell^{\prime}}\right\}=\left[m\left(\ell^{\prime}+1\right)-m^{\prime}(\ell+1)\right] v_{m+m^{\prime}}^{\ell+\ell^{\prime}}
$$

This includes a Virasoro symmetry generated by the operators $L_{n}=v_{n}^{0}$, whose Poisson brackets obey the algebra

$$
\left\{L_{n}, L_{m}\right\}=(m-n) L_{m+n},
$$

which is a subalgebra of the $w_{\infty}$ algebra (3.4).

We suggested in [10] that the infinite set of charges appearing in the quantum version of the $w_{\infty}$ symmetry should be considered as an infinite set of discrete hair (termed W-hair) that is responsible for the maintenance of quantum coherence for the two-dimensional stringy black hole, since the corresponding quantum-gravity scattering matrix, obtained from correlation functions of marginal world-sheet vertex operators, is invariant under these symmetries.

\footnotetext{
${ }^{4}$ These symmetries were first discovered in operator product expansions of vertex operators corresponding to the discrete stringy 'tachyon' states of the two-dimensional (target space) $c=1$ Liouville string theory [26] that, from a target space-time point of view, is the asymptotic limit of the Euclidean black hole of [4].
} 
Consider, for example, the propagation of a 'tachyon', which is a massless particle in two dimensions. In flat space it is associated with the vertex operator:

$$
\phi_{-1 / 2,0,0}^{c,-c}=\left(g_{++} g_{--}\right)^{-\frac{1}{2}} F\left(\frac{1}{2} ; \frac{1}{2} ; 1 ; \frac{g_{+-} g_{-+}}{g_{++} g_{--}}\right),
$$

where $F$ denotes a hypergeometric function and $g_{a b}, a, b=+,-$ represent the components of a generic $S L(2, R)$ element. The operator (3.6) is exactly marginal in a two-dimensional flat-space string theory.

However, this is not the case in the background of a two-dimensional space-time black hole (Euclidean or Minkowski, the latter being obtained by analytic continuation of the compact variable ('temperature') in the cigar metric of [4]). In this case, the corresponding exactly marginal operator is [6]

$$
L_{0}^{1} \bar{L}_{0}^{1}=\phi_{-1 / 2,0,0}^{c,-c}+i\left(\psi^{++}-\psi^{--}\right)+\ldots
$$

where

$$
\psi^{ \pm \pm} \equiv:\left(\bar{J}^{ \pm}\right)^{N}\left(J^{ \pm}\right)^{N}\left(g_{ \pm \pm}\right)^{j+m-N}:
$$

with $J^{ \pm} \equiv(k-2)\left(g_{ \pm \mp} \partial_{z} g_{ \pm \pm}-g_{ \pm \pm} \partial_{z} g_{ \pm \mp}\right)$, and $\bar{J}^{ \pm} \equiv(k-2)\left(g_{\mp \pm} \partial_{z} g_{ \pm \pm}-g_{ \pm \pm} \partial_{\bar{z}} g_{\mp \pm}\right)$, where $k$ is the WZ model level parameter [4]. The combination $\psi^{++}-\psi^{--}$generates a level-one massive string mode, and the dots in equation (3.7) represent operators that generate higher-level massive string states ${ }^{5}$. As discussed in [10], these modes are solitonic, with fixed energy and momentum. As such, they are completely de-localized in space-time.

Since the flat-space 'tachyon' vertex operator (3.6) is not exactly marginal in a black-hole background, the corresponding RG $\beta$ function is non-vanishing and hence, by (2.14), the entropy associated with tachyonic 'matter' increases inexorably, i.e, information is lost, if the higher-level string modes in (3.7) are neglected. Conversely, if these string modes are taken into account, the corresponding RG $\beta$ function vanishes, entropy does not increase with the world-sheet RG flow, which we identify with the target-space temporal time flow in our approach. Thus, there is no information loss: it is stored by the higher-level string modes.

In order to guarantee the exact marginality of the corresponding vertex operator (3.6), topological states must be included in the scattering matrix of strings in a two-dimensional black-hole background. These topological modes are not detectable in a local scattering experiment, leading to an apparent 'loss' of quantum coherence, which is an artefact of the phenomenological truncation of the scattering process within a local effective field theory (LEFT) framework. Associated with this apparent 'loss' of quantum coherence there is an apparent 'increase' in entropy at a rate quantified by the right-hand-side of (2.14), since the truncated $\mathrm{RG} \beta^{i}$ functions of the non-marginal propagating modes do not vanish.

Nevertheless, the conserved W-hair charges are in principle measurable, and ways for doing so in principle have been outlined in [12]. These are reviewed in the next Section, where we also present arguments for the elevation of the W-hair to four-dimensional space times. In this case,

\footnotetext{
${ }^{5}$ Another example of an exactly-marginal operator is $L_{0}^{2} \bar{L}_{0}^{2}=\psi^{++}+\psi^{--}+\psi^{-+}+\psi^{+-}+\ldots$, which also involves in an essential way operators for massive string modes. The coupling corresponding to this world-sheet deformation of the coset model is associated with a global rescaling of the target space-metric [6], and therefore to a global constant shift of the dilaton field. Thus it produces shifts in the black hole mass [4].
} 
the area-preserving property of the $W_{\infty}$ symmetry becomes important for preserving the area of the two-dimensional surface of the black-hole horizon.

\section{Elevation to Four Dimensions}

We have argued in [12] that the coset singularity structure of the two-dimensional stringy black hole and generic properties of its associated discrete states have counterparts for sphericallysymmetric black-hole configurations in four space-time dimensions. We now review the basic arguments supporting this conjecture, which have been reinforced by subsequent formal developments.

\subsection{Embedding of the Two-Dimensional Black Hole}

We consider a string theory with a spherically-symmetric gravitational background of blackhole type, which is a solution of the Einstein equations, generalised to the effective field theory derived from string theory. The metric tensor is given by an Ansatz of the form:

$$
d s^{2}=g_{\alpha \beta} d x^{\alpha} d x^{\beta}+e^{W(r, t)} d \Omega^{2},
$$

where $\mathrm{W}(\mathrm{r}, \mathrm{t})$ is a non-singular function, $x^{\alpha, \beta}$ denote the $r, t$ coordinates, and $d \Omega^{2}=d \theta^{2}+\sin ^{2} \theta d \phi^{2}$ denotes the line element on a spherical surface that does not change with time.

We remind the reader that in pure gravity all the classical spherically-symmetric solutions to the equations of motion obtained from higher-derivative gravitational actions with an arbitrary number of curvature tensors are static [31], and that a similar result holds for stringy black holes at tree level. The standard Schwarzschild solution of the spherically-symmetric four-dimensional black hole can be put in the form (4.1) by an appropriate transformation of variables.

We consider the Schwarzschild solution in Kruskal-Szekeres coordinates [32]

$$
d s^{2}=-\frac{32 M^{3}}{r} e^{-\frac{r}{2 M}} d u d v+r^{2} d \Omega^{2},
$$

where $r$ is a function of $u, v$, given by

$$
\left(\frac{r}{2 M}-1\right) e^{\frac{r}{2 M}}=-u v .
$$

Note that, although the two-dimensional metric components depend on the variables $u, v$, the black hole solution is nevertheless static. Changing variables to

$$
\begin{gathered}
e^{-\frac{r}{4 M}} u=u^{\prime}, \\
e^{-\frac{r}{4 M}} v=v^{\prime}
\end{gathered}
$$

and taking into account the Jacobian $J$ of the transformation of the area element $d u d v$, we can put the two-dimensional metric in the form

$$
g_{b h}\left(u^{\prime}, v^{\prime}\right)=\frac{e^{D\left(u^{\prime}, v^{\prime}\right)} d u^{\prime} d v^{\prime}}{1-u^{\prime} v^{\prime}}
$$

with the scale factor being given by $16 M^{2} e^{-\frac{r^{\prime}\left(u^{\prime}, v^{\prime}\right)}{2 M}} J\left(u^{\prime}, v^{\prime}\right)$, where $r^{\prime}$ is the coordinate $r$ re-expressed in therms of the coordinates $u^{\prime}, v^{\prime}$. 
The metric (4.5) is a conformally-rescaled form of Witten's two-dimensional black hole solution [4]. Since the latter is described by an exact conformal field theory, the same is true after this conformal rescaling. From a $\sigma$-model point of view, this rescaling simply expresses a change of renormalisation scheme ${ }^{6}$. The global properties, such as singularities, remain unchanged from the two-dimensional string case.

\subsection{Discrete Topological States}

In particular, the infinite-dimensional W-symmetry associated with the $\mathrm{SU}(1,1) / \mathrm{U}(1)$ coset structure of the dilaton-graviton sector in the two-dimensional model is elevated to become a model-independent feature of spherically-symmetric four-dimensional string configurations. Such structures are intimately connected with the existence of topological solitonic non-propagating states. These states are essentially spherically-symmetric solutions of the low-energy equations of motion obtained from the string theory in manifolds with topology $\mathrm{SU}(1,1) / \mathrm{U}(1) \times \mathscr{M}^{2}$, where $\mathscr{M}^{2}$ is a two-dimensional manifold of constant curvature. They are associated with jumps in the number of degrees of freedom at discrete values of energy and momentum as a result of relaxation of certain gauge theory constraints, as shown below. The simplest example is where $\mathscr{M}^{2}=S^{2}$, the sphere, which describes the spherically-symmetric four-dimensional black hole solution of interest to us here. The associated infinity of discrete topological (non-propagating) states, with definite energies and momenta, couple to the massless propagating 'tachyon' string matter and thereby ensure conformal invariance of the associated $\sigma$-model action, as described above for the purely two-dimensional stringy black hole of [4].

The infinity of discrete topological states in a $D$-dimensional target-space string theory are similar in nature to those of the two-dimensional case [33, 26]. These states can be seen via the gauge conditions for a rank- $n$ tensor multiplet:

$$
D^{\mu_{1}} A_{\mu_{1} \mu_{2} \ldots \mu_{n}}=0
$$

where $D_{\mu}$ is a (curved-space) covariant derivative. To illustrate our arguments, consider the simplified case of weak gravitational perturbations around flat space, with a linear dilaton field of the form $\Phi(X)=Q_{\mu} X^{\mu}$, in which case the Fourier transform of (4.6) is

$$
(p+Q)^{\mu_{1}} \tilde{A}(k)_{\mu_{1} \mu_{2} \ldots \mu_{n}}=0
$$

We observe that there is a jump in the number of degrees of freedom at the discrete momentum $p=-Q$. The fixed momentum corresponds to complete uncertainty in space, so such states are delocalised, and can be considered as quasi-topological and non-propagating soliton-like states. In ordinary string theories, such states carry a small statistical weight, due to the continuous spectrum of the various string modes. However, when strings propagate in spherically-symmetric fourdimensional background space-times, these discrete states assume particular importance. Such backgrounds are effectively two-dimensional, and therefore all the transverse modes of higherrank tensors can be gauged away using Ward identities of the form (4.6), except for the topological

\footnotetext{
${ }^{6}$ The function $D(u, v)$ can be regarded also as a part of the two-dimensional dilaton in the given renormalisation scheme.
} 
modes. In the case of four-dimensional spherically-symmetric black holes, these $s$-wave topological modes constitute the final stages of their evaporation [10], and assume responsibility for the maintenance of quantum coherence $[10,12]$.

\subsection{Phase-Space-Area-Preserving $w_{\infty}$ Symmetries}

In another example [9], a $w_{\infty}$ symmetry arises in the phase space of matter in a four-dimensional extremal solitonic black hole background in the context of $N=2, D=4$ supergravity. This is a BPS solution that interpolates between a maximally-supersymmetric $\mathrm{AdS}_{4}$ space-time at large radial distances and $\mathrm{AdS}_{2} \times H^{2}$, where $\mathrm{AdS}_{2}$ refers to the radial-coordinate/time part of the spacetime and $H^{2}$ refers to the angular part of the space-time, which is a hyperbolic two-dimensional manifold of constant curvature. The $\mathrm{AdS}_{2} \times H^{2}$ geometry characterises the space-time near the horizon of the black hole. The analysis of [9] showed that the dynamics of a quantum-mechanical massive particle with non-trivial magnetic charge in the near-horizon geometry is described effectively by a one-spatial-dimensional Hamiltonian $H$, characterised by a $w_{\infty}$ symmetry that preserves the two-dimensional phase-space area symplectic form $\Omega=d p \wedge d q-d H \wedge d t$, with $q$ the spatial coordinate, $p$ the canonical momentum and $t$ the time. The energy spectrum of this particle is continuous and bounded from below: $E>0$, but the ground state is non-normalizable, with an infrared (IR) divergence, which was regularised in [9] by putting the system in a box. The IR-regularised system is also invariant under a $w_{\infty}$ that contains a Virasoro symmetry (3.5), which can be associated with the asymptotic symmetries of the $\mathrm{AdS}_{2}$ space time, i.e., the diffeomorphisms that leave invariant the $\mathrm{AdS}_{2}$ metric, whose quantum version includes a central extension. Such asymptotic symmetries are symmetries of the quantum-gravity scattering matrix for the full four-dimensional $\mathrm{AdS}_{2} \times H^{2}$ extremal black hole of [9] ${ }^{7}$.

Hence, the particle system is characterised by an infinity of conserved charges of the $v_{m}^{\ell}$ type (3.3), in which the rôle of the $x, y$ coordinates is played by appropriate combinations of the phasespace coordinates of the particle [9], and hence is completely integrable. From our point of view, the presence of an infinity of conserved quantities for the particle in the near-horizon geometry of the black hole also guarantees quantum coherence, in the sense that the infinity of conserved charges $v_{m}^{\ell}$, which remain constant during the scattering of matter off the black-hole background, retain information during the evaporation of the latter. The situation of the coherence-preserving $w_{\infty}$ algebra is exactly analogous to that preserving the phase-space area for a massless 'tachyonic' string matter in the two-dimensional stringy black hole - or its four-dimensional extension with topology $\mathrm{SU}(1,1) / \mathrm{U}(1) \times S^{2}$ - as discussed above.

As discussed above, the elevation of such classical phase-space-area- $w_{\infty}$ symmetry algebras to fully quantum coherence-preserving algebras necessarily involves discrete topological states of the string. In two dimensions, as we have seen, the latter mix with the propagating massless matter states in order to guarantee the conformal invariance of the corresponding vertex operators in the presence of a stringy black hole background [10], and hence preserve quantum coherence according to the general arguments of Section 2.

\footnotetext{
${ }^{7}$ An asymptotic symmetry of the quantum-gravity scattering matrix under supertranslations of generic black hole backgrounds has been examined in $[14,15]$.
} 


\subsection{W-hair and Quantum Coherence}

As discussed in [13], each of these discrete solitonic states can be represented as a singular gauge configuration, whose conserved $W$-charges can be measured in principle by generalized Aharonov-Bohm phase effects. Moreover, the topological higher-spin string states leave their imprint via selection rules in the scattering matrix, where they appear as (resonance) poles, corresponding to discrete energies and momenta and leading to certain selection rules. In the stringy black hole case, there is an infinite set of such black hole soliton states, classified by the quadratic Casimir and 'magnetic' quantum numbers of an internal symmetry group [13], which are excited at calculable energies and decay into distinctive combinations of light final-state particles.

The stringy scattering matrix is, in general, well defined in the presence of such black-hole backgrounds, since the world-sheet correlation functions among the appropriate exactly marginal vertex operators are unitary. This is because, as mentioned previously, in addition to the parts corresponding to the propagating string states, these operators contain an infinity of topological non-propagating states. In practice, scattering experiments in the laboratory, which involve a finite number of localised (in spacetime) particle states, cannot detect the delocalised states. Hence, from the point of view of a local low-energy observer, there would be an apparent decoherence, although this would not entail any pathologies in the full stringy theory of quantum gravity.

\section{Phase-Space vs Horizon-Area-Preserving $W$ Symmetries}

We now explore the potential relation between the quantum $W_{1+\infty}$ algebras that are symmetries of the stringy quantum gravity S-matrix and the classical area-preserving symmetries that preserve the horizon area of a classical (non-evaporating) black hole and hence its entropy. This relation is subtle, and at present is not understood in its full generality, at least by the authors. Nevertheless, as we shall discuss below, by representing the horizon of the black hole as a thick D(irichlet) brane, such a relation becomes evident. As a prelude to this result, we first discuss the case of an infinitely-coloured four-dimensional black hole in a $\mathrm{SU}(N \rightarrow \infty)$ Yang-Mills gauge theory, which has an infinite amount of gauge hair, as allowed by the 'no-hair' theorem [30].

\subsection{Black Holes with Infinitely-Coloured Hair}

As we have discussed, the classical $w_{\infty}$ algebra preserves the two-dimensional area of an 'internal space' with the topology of a sphere [24, 25]. The issue is whether the 'internal' sphere can be identified with the real horizon of the spherically-symmetric four-dimensional Schwarzschild black hole. To address this question, we consider examples of four-dimensional spherically-symmetric black holes with infinitely-coloured hair, which realize explicitly a classical $w_{\infty}$. These appear in an effective field theory example of a black-hole solution in $\mathrm{SU}(N \rightarrow \infty)$ gauge theory in a four-dimensional AdS space-time with negative cosmological constant, which plays the role of a regulator for the black-hole solution that makes it well-defined [30]. This anti-de-Sitter (AdS) regulator was given physical significance via the AdS/CFT bulk/boundary correspondence, and turns out to be physically important, as we argue below. For the present discussion, the interest of these 
black holes with black holes with infinitely coloured hair is that classically there is an isomorphism between $\mathrm{SU}(N \rightarrow \infty)$ and $w_{\infty}[24,28]^{8}$.

To develop this point, consider a unit sphere $S^{2}$ with coordinates $\theta, \phi$ and the quantities:

$$
x_{1}=\sin \theta \cos \phi, \quad x_{2}=\sin \theta \sin \phi, \quad x_{3}=\cos \theta, \quad \text { with } \quad \sum_{i=1}^{3} x_{i}^{2}=1 .
$$

The spherical harmonics $Y_{\ell m}(\theta, \phi)$ are harmonic polynomials of degree $\ell$ in $x_{i}$ :

$$
Y_{\ell m}(\theta, \phi)=\sum_{i_{k}=1,2,3,3_{k=1, \ldots, \ell}} \alpha_{i_{1} \ldots i_{\ell}}^{(m)} x_{i_{1}} \ldots x_{i_{\ell}} .
$$

For fixed $\ell$ there are $2 \ell+1$ linearly independent symmetric and traceless tensors $\alpha_{i_{1} \ldots i_{\ell}}^{(m)}, m=$ $-\ell, \ldots, \ell$. Let us consider an $\mathrm{SU}(2)$ subgroup of the $\mathrm{SU}(\mathrm{N})$ group in the limit $N \rightarrow \infty$, generated by $S_{i}$ with standard commutation relations

$$
\left[S_{i}, S_{j}\right]=i \varepsilon_{i j k} S_{k}
$$

From the standard theory of angular momentum [34, 29], we know that a representation of the $N^{2}-1$ generators of the group $\mathrm{SU}(\mathrm{N})$ can be expressed as follows in terms of the $S_{i}$ matrices and the $\alpha$-tensors in (5.2):

$$
\begin{aligned}
S_{\ell, m}^{(N)} & =\sum_{i_{k}=1,2,3,3_{k=1, \ldots, \ell}} \alpha_{i_{1} \ldots i_{\ell}}^{(m)} S_{i_{1}} \ldots S_{i_{\ell}} \\
{\left[S_{\ell, m}^{(N)}, S_{\ell^{\prime}, m^{\prime}}^{(N)}\right] } & =i f_{\ell, m ; \ell^{\prime}, m^{\prime}}^{(N) \ell^{\prime \prime}, m^{\prime \prime}, S^{\prime \prime}, m^{\prime \prime}} .
\end{aligned}
$$

Upon the rescaling

$$
S_{i} \rightarrow T_{i} \equiv \frac{2}{N} S_{i}
$$

we arrive at bounded matrix elements as $N \rightarrow \infty$ : $\left|\left(T_{i}\right)_{b}^{a}\right| \leq 1$, with the well-defined commutator algebra

$$
\left[T_{i}, T_{j}\right]=i \frac{2}{N} \varepsilon_{i j k} T_{k} \rightarrow 0, N \rightarrow \infty
$$

and the Casimir element

$$
T^{2}=\sum_{i=1}^{3} T_{i}^{2}=1-\frac{1}{N} \rightarrow 1, N \rightarrow \infty .
$$

We conclude from (5.5) and (5.6) that in the $N \rightarrow \infty$ limit, a representation of the (commuting) generators of the $S U(2)$ subgroup of $S U(\infty)$ is provided by the quantities $x_{i}$ in (5.1). If one considers any two functions of $x_{i}$ on a spherical surface, $f\left(x_{1}, x_{2}, x_{3}\right), g\left(x_{1}, x_{2}, x_{3}\right)$, each of which can be expanded in terms of the spherical harmonics (5.2), (5.5) shows that in the limit $N \rightarrow \infty$ the corresponding matrix polynomials of the generators $f\left(T_{1}, T_{2}, T_{3}\right)$ and $g\left(T_{1}, T_{2}, T_{3}\right)$ satisfy

$$
\frac{N}{2 i}[f, g] \rightarrow \varepsilon_{i j k} \frac{\partial f}{\partial x_{j}} \frac{\partial g}{\partial x_{k}}, \quad N \rightarrow \infty .
$$

\footnotetext{
${ }^{8}$ For SU(N) gauge theories with finite $N$, the geometry of the corresponding space is non-commutative [29], the commutativity being restored in the limit $N \rightarrow \infty$.
} 
Replacing the SU(2) generators in (5.3) by the rescaled ones (5.4), one finds for the $N^{2}-1$ matrices $T_{\ell m}^{(N)}$ :

$$
\frac{N}{2 i}\left[T_{\ell, m}^{(N)}, T_{\ell^{\prime}, m^{\prime}}^{(N)}\right] \rightarrow \quad\left\{Y_{\ell, m}, Y_{\ell^{\prime}, m^{\prime}}\right\}, \quad N \rightarrow \infty .
$$

The Poisson (classical) algebra of the spherical harmonics is known to be that of the infinitedimensional area-preserving diffeomorphisms on the sphere $\operatorname{SDiff}\left(S^{2}\right)$ :

$$
\begin{aligned}
\left\{Y_{\ell, m}, Y_{\ell^{\prime}, m^{\prime}}\right\} & =\frac{M\left(\ell+\ell^{\prime}-1, m+m^{\prime}\right)}{M(\ell, m) M\left(\ell^{\prime}, m^{\prime}\right)}\left(\ell^{\prime} m-\ell m^{\prime}\right) Y_{\ell+\ell^{\prime}-1, m+m^{\prime}} \\
& +\sum_{n=1} g_{2 n}\left(\ell, \ell^{\prime}\right) C_{\ell, m, \ell^{\prime}, m^{\prime}}^{\ell+\ell^{\prime}-1-2 n, m+m^{\prime}} Y_{\ell+\ell^{\prime}-1-2 n, m+m^{\prime}}
\end{aligned}
$$

where the $M, g_{2 n}$ are normalization functions and the structure constants $C$ are given in the fourth paper in [24]. This algebra is known to be isomorphic to the classical area-preserving $w_{\infty}$ algebra.

Since the classical gauge fields of the $\mathrm{SU}(N \rightarrow \infty)$ gauge theory can be expanded in the basis of the matrices $T_{\ell, m}^{(N)}$, the above considerations, and in particular (5.8), indicate that in this example of an infinitely-coloured gauge black hole, this area-preserving diffeomorphism symmetry preserves the horizon area, once we identify the 'internal' sphere $S^{2}$ with the actual horizon sphere of the spherically-symmetric $\mathrm{SU}(\infty)$ black hole. In this case, the entropy of the black hole can be preserved classically by the $w_{\infty}$ hair. If one views this $\mathrm{SU}(\infty)$ gauge theory as a low-energy limit of some string theory then, in view of our world-sheet renormalization-group interpretation of the target time that leads to (2.14), the conservation of the classical area should correspond to the conformal invariance of the corresponding world-sheet, which guarantees the vanishing of the right-hand-side of (2.14) through the zeroes of the $\beta^{i}$ functions of appropriate combinations of the couplings $g^{i}$. In the case at hand, the set $\left\{g^{i}\right\}$ consists of the graviton $G_{\mu v}$ and $\mathrm{SU}(\infty)$ gauge field background modes: $A_{\mu}^{a}, a=1 \ldots \infty$.

Hence one can understand the entanglement of the massless (propagating) graviton states in this four-dimensional extremal black hole with the discrete infinity of gauge states by analogy with the entanglement of the propagating massless 'tachyonic' matter with the infinity of the discrete massive states in the two-dimensional black hole case, $(3.6,3.7)$, as follows.

Upon embedding the $\mathrm{SU}(\infty)$ gauge theory in a string model with propagating graviton and gauge field backgrounds, one deforms the corresponding $\sigma$-model by adding to the usual graviton (spin-2) deformations the following vector deformation of the gauge field $A_{\mu}^{a}$ :

$$
\mathscr{Z}=\int[D X] e^{\frac{1}{2 \pi \alpha^{\prime}} \int_{\Sigma} \sqrt{\gamma} \partial_{\alpha} X^{\mu} \partial^{\alpha} X^{v} G_{\mu v}(X)} \operatorname{Tr}\left(e^{\int_{\partial \Sigma} A_{\mu}^{a} t^{a} \partial_{\tau} X^{\mu}}\right) .
$$

Here we use the standard notation for a $\sigma$-model propagating on a world-sheet $\Sigma$ with a boundary $\partial \Sigma$ (to accommodate open strings corresponding to gauge field excitations), describing the motion of a string in a target-space with coordinates $X^{\mu}$ and a metric background $G_{\mu v}(X)$. The trace $\operatorname{Tr}$ is over colour indices, and the $t^{a}$ are the generators of the $\mathrm{SU}(\infty)$ colour group.

The presence of an AdS background with a non-vanishing cosmological constant $\Lambda<0$ as a regulator for the black hole of [30] implies that, on embedding such a theory in a string theory, the graviton world-sheet $\beta$-function, $\beta_{\mu \nu}^{G}$, is no longer zero. Indeed, a $\sigma$-model one-loop analysis, which suffices for the weak gravity in the near-horizon black-hole geometry that we consider here, 
is given by the target-space Ricci tensor:

$$
\beta_{\mu \nu}^{G}=R_{\mu \nu},
$$

which for an anti de Sitter space time reads

$$
R_{\mu \nu}^{G}=\Lambda g_{\mu v} \neq 0, \Lambda<0 .
$$

If this divergence were not cancelled against other background fields, so as to restore marginality of the corresponding world-sheet operator, then there would be entropy increase under the RG flow, which in our approach is identified with real-time flow (2.14), and thus information loss in the classical stationary black-hole background.

The presence of an infinity of gauge fields can provide a resolution to this problem with the infinity of zero-momentum gauge field modes $A_{\mu}^{a}, a=1, \ldots N \rightarrow \infty$ condensing:

$$
\left\langle\sum_{a=1}^{\infty} F_{\mu v}^{a} F^{a \mu v}\right\rangle \neq 0
$$

The infinite number of colours plays a crucial rôle in guaranteeing a macroscopic occupation of the quantum-mechanical ground state of this system, which is a prerequisite for the formation of a quantum condensate. One needs delocalised zero modes, because they are constant in space-time, and hence their condensation guarantees the space-time translational invariance of the condensate. In string theory there is an infinity of higher-order self-interactions among the gauge fields in the low energy string effective action, which can lead to the formation of such a condensate.

Coupling gauge fields and gravitons, the corresponding graviton $\beta$-function (5.11) is modified in the presence of such a condensate (5.12) to

$$
\beta_{\mu \nu}^{G}=\Lambda g_{\mu v}+\frac{1}{2} V\left(\left\langle F_{\mu \nu}^{a} F_{\mu \nu}^{a}\right\rangle\right) g_{\mu \nu},
$$

where $V\left(\left\langle F_{\mu \nu}^{a} F_{\mu \nu}^{a}\right\rangle\right)$ is the scalar vacuum energy arising from the condensate. The structure on the right-hand side of (5.13) is the only one consistent with Lorentz invariance of the vacuum. The form of $V\left(\left\langle F_{\mu \nu}^{a} F_{\mu \nu}^{a}\right\rangle\right)$ is, to lowest order in the field strengths:

$$
V\left(\left\langle F_{\mu \nu}^{a} F_{\mu \nu}^{a}\right\rangle\right) \propto\left\langle F_{\mu \nu}^{a} F_{\mu \nu}^{a}\right\rangle+\ldots>0,
$$

where the ... indicate higher derivative terms that are present in string theory and are essential in providing the necessary self-interactions among the non-Abelian gauge fields to guarantee the formation of condensates.

We observe from (5.14) and (5.13) that a cancellation of the right-hand-side is possible because $\Lambda<0$ for AdS backgrounds. Thus we recover a conformal graviton background in the presence of a condensate formed by the delocalised gauge states. The latter realize a $W_{\infty}$ algebra, which preserves classically the horizon area of the black hole, thus providing a picture of the area-preserving nature of the W-hair that is consistent with the conformally-invariant mixing of graviton states with the infinity of gauge states. 


\subsection{Horizons as 'Thick' D(irichlet)-Branes and SU( $\infty$ ) Gauge Theory}

If we represent the horizon of the four-dimensional black hole as a two-brane, then we immediately face the problem of recoil once a string matter state, represented by a closed or open string, encounters the horizon surface. If it is a closed string, it may split into two open strings (to preserve the chirality of the state), whereas if it is an open string then at least one of its ends will be attached to the horizon, causing the latter to recoil in order to conserve momentum. The recoil of the horizon induces local fluctuations on the horizon that can be studied using logarithmic conformal field theory on the world-sheet [35, 36], that have also been argued to carry information [37]. A fluctuating (recoiling) horizon may be represented (from the point of view of a low-energy observer) as a 'thick' D-brane stack of $N \rightarrow \infty$ concentric branes. For macroscopic black holes, with large horizons compared to the wavelength of the infalling matter, such concentric branes may be well approximated locally by a stack of parallel flat $N \rightarrow \infty$ branes. Such constructions are equivalent to $\mathrm{SU}(N \rightarrow \infty)$ gauge theories [38], as can be seen intuitively by considering the topologicallyequivalent ways ( $N^{2}-1$ for $\mathrm{SU}(\mathrm{N})$ gauge theory) in which an open string can be attached to a stack of $N$ parallel D-branes.

When infalling matter crosses the horizon of such a thick-horizon-brane black hole, the recoil is described by open string excitations that carry the $S U(\infty)$ charges, leading to the infinite hair of the black hole and corresponding to the horizon-area preserving $w_{\infty}$ symmetry discussed previously in Subsection 5.1. The important aspect of this example is that now the $S U(\infty)$ symmetry is also a coherence-preserving symmetry of the associated quantum-gravity scattering matrix in the presence of the $\mathrm{SU}(\infty)$ black hole.

\subsection{Two-Dimensional $W_{\infty}$ Symmetries as Gauged Four-Dimensional Symmetries}

In this subsection we speculate on a potential generalization of the above result to arbitrary four-dimensional space-times with space-time singularities having the (stringy-black-hole-like) structure $\mathrm{SU}(1,1) / \mathrm{U}(1) \otimes S^{2}$ or, more generally, embedding two-dimensional singularity structures admitting $W_{\infty}$ symmetries into spherically-symmetric four-dimensional space-times with constant curvature, as in the example of the four-dimensional black-hole soliton of [9].

Our starting point is the construction [25] of $W_{\infty}$ (and $w_{\infty}$ ) gauge theories in terms of $(d+2)$ dimensional local fields, where $d$ is the dimension of space-time on which the algebras live: $d=2$ in the case of interest to us. One can define the $W_{\infty}$ quantum algebra as a commutator algebra of Hermitian operators $\xi\left(a, a^{\dagger}\right)$, where $a, a^{\dagger}$ are the harmonic-oscillator annihilation and creation operators. One may parametrize the operators $\xi\left(a, a^{\dagger}\right)$ using coherent states:

$$
: \xi\left(\hat{a}, \hat{a}^{\dagger}\right):=\int d^{2} z e^{-|z|^{2}}|z>\xi(z, \bar{z})<z|
$$

where $\left|z>=e^{\hat{a}^{\dagger} z}\right| 0>,<z|=<0| e^{\hat{a} z},\left\langle z^{\prime}\left|z>=e^{z^{\prime} z}, \hat{a}\right| z>=z|z>,<z| \hat{a}^{\dagger}=<z\right| \bar{z}$, and the normalization condition is $\int d^{2} z e^{-|z|^{2}}|z><\bar{z}|=1$, with $d^{2} z \equiv \frac{1}{\pi} \operatorname{Re} z \operatorname{Im} z$, and the $: \xi\left(\hat{a}, \hat{a}^{\dagger}\right):$ is an (anti-)normal-ordered operator, where the creation operators are always placed to the right of the annihilation operators. 
The coordinates $z, \bar{z}$ are viewed in [25] as a group-theoretical ('colour') space. Introducing a gauge potential $A_{\mu}\left(x, \hat{a}, \hat{a}^{\dagger}\right)$, where $\mu=1, \ldots d$ is a $d$-dimensonal space time $\{x\}$ index,

$$
\hat{A}_{\mu}(x) \equiv A_{\mu}\left(x, \hat{a}, \hat{a}^{\dagger}\right)=\int d^{2} z e^{|z|^{2}}\left|z>A_{\mu}(x, z, \bar{z})<z\right|
$$

and infinite-dimensional set of infinitesimal $W_{\infty}$ gauge transformations can be introduced as follows:

$$
\delta \hat{A}_{\mu}(x)=\partial_{\mu} \hat{\xi}(x)+i\left[\hat{\xi}(x), \hat{A}_{\mu}(x)\right], \quad \delta A_{\mu}(x, z, \bar{z})=\partial_{\mu} \xi(x, z, \bar{z})-\left\{\left\{\xi, A_{\mu}\right\}\right\}_{\text {Moyal }}(x, z, \bar{z}),
$$

where $\{\{., .\}\}_{\text {Moyal }}$ denotes a Moyal bracket, defined as

$$
\left\{\left\{\xi_{1}, \xi_{2}\right\}\right\}_{\text {Moyal }}(z, \bar{z}) \equiv i \sum_{n=1}^{\infty} \frac{(-1)^{n}}{n}\left(\partial_{z}^{n} \xi_{1}(z, \bar{z}) \partial_{\bar{z}}^{n} \xi_{2}(z, \bar{z})-\partial_{\bar{z}}^{n} \xi_{1}(z, \bar{z}) \partial_{z}^{n} \xi_{2}(z, \bar{z})\right) .
$$

In this construction, the generators of $W_{\infty}, \rho[\xi]$, are linear functionals of $\xi(z, \bar{z})$ and satisfy at a quantum level [25]:

$$
\left[\rho\left[\xi_{1}\right], \rho\left[\xi_{2}\right]\right]=i \rho\left[\left\{\left\{\xi_{1}, \xi_{2}\right\}\right\}_{\text {Moyal }}\right] .
$$

The classical area-preserving $w_{\infty}$ Lie algebra, obtained from $W_{\infty}$ by an appropriate contraction discussed in [25], is then

$$
\left[\rho\left[\xi_{1}\right], \rho\left[\xi_{2}\right]\right]=i \rho\left[\left\{\xi_{1}, \xi_{2}\right\}_{\text {Poisson }}\right],
$$

where $\{., .\}_{\text {Poisson }}$ denotes the (classical) Poisson bracket.

Notice that, in this representation, the $W_{\infty}$ gauge fields $A_{\mu}(x, z, \bar{z})$ are defined in a $d+2$ dimensional space time $\{x, z, \bar{z}\}$ with a two-dimensional 'internal' space spanned by the $\{z, \bar{z}\}$ coordinates. The Yang-Mills-type $\mathscr{S}$ action, which is invariant under the $W_{\infty}$ gauge transformations, has the form (5.16),

$$
\mathscr{S}=-\frac{1}{4 g^{2}} \int d^{d} x \frac{1}{4} \operatorname{Tr}\left(\hat{\mathscr{F}}_{\mu v} \hat{\mathscr{F}}^{\mu v}\right): \hat{\mathscr{F}}_{\mu v}=\partial_{\mu} \hat{A}_{v}(x)-\partial_{v} \hat{A}_{\mu}(x)-i\left[\hat{A}_{\mu}, \hat{A}_{v}\right],
$$

where $g$ is a coupling constant, and can be rewritten using the coherent-state representation as [25]:

$$
\begin{aligned}
\mathscr{S} & =-\frac{1}{4 g^{2}} \int d^{d} x d^{2} z \sum_{n=0}^{\infty} \frac{(-1)^{n}}{n} \partial_{z}^{n} \mathscr{F}_{\mu v}(x, z, \bar{z}) \partial_{\bar{z}}^{n} \mathscr{F}^{\mu v}(x, z, \bar{z}): \\
\mathscr{F}_{\mu \nu} & =\partial_{\mu} A_{v}(x, z, \bar{z})-\partial_{v} A_{\mu}(x, z, \bar{z})+\left\{\left\{A_{\mu}, A_{v}\right\}\right\}_{\text {Moyal }}(x, z, \bar{z}) .
\end{aligned}
$$

The reader should notice the non-local nature of the action in terms of the $z, \bar{z}$ variables. In fact, as stressed in [25], it is this non-local nature of the action that differentiates the $W_{\infty}$ from $w_{\infty}$ as far as the association with the $S U(\infty)$ gauge theory is concerned. It is the spectrum of the $W_{\infty}$ that can be viewed as the $N \rightarrow \infty$ limit of $S U(N)$, not that of the $w_{\infty}$.

Thus we see that, in analogy with the $S U(N \rightarrow \infty)$ example discussed previously, the group Trace (here over the infinite-dimensional $W$-algebra) is replaced by an integral over the coordinates of the two-sphere $S^{2}$. In fact, in the action (5.21) there are no exponentially-damped $e^{-|z|^{2}}$ factors so, in order to have well-defined expressions upon partial integration, one must require the fields and their derivatives to vanish at $z, \bar{z} \rightarrow \pm \infty$. For the case of the sphere, there is no boundary, 
and hence one is not facing this problem. One should identify the (non-compact) variables $z, \bar{z}$ with some stereographic-projection coordinates of the $S^{2}$. In the spirit of (5.8), then, one may expect that the infinite-dimensional $W_{\infty}$ gauge symmetry indeed corresponds to an area-preserving symmetry that leaves invariant the area of $S^{2}, \operatorname{SDiff}\left(S^{2}\right)$.

For the stringy black hole of interest, the above considerations apply if we identify the gauged $W_{\infty}$ symmetry with the $W_{1+\infty}$ of the string states discussed previously, which involves discrete delocalised states. It has been shown in [39] that, for the asymptotically-flat two-dimensional string theory, i.e., the $c=1$ Liouville model, for each of these states one can construct 'discrete gauge states', at various mass string levels corresponding to tensorial gauge fields, which satisfy the same $W_{\infty}$ algebra as the topological discrete states of the two-dimensional string theory [26]. These states carry the $W_{1+\infty}$ charges and thus one can gauge the $W_{\infty}$ algebras by coupling them to the corresponding currents. In this way one may have an explicit realisation of the gauge transformations (5.16) for the two-dimensional string. The generalization to the curved black-hole background is a non-trivial task, since one expects a mixing of various mass levels in the exactly-marginal vertex operators describing the discrete gauge states, as with the standard topological states.

In fact there is a much simpler formal picture that describes the situation non-perturbatively in the string coupling $[4,40]$. Scattering theory around the particular solution of string theory that describes the asymptotic state of the two-dimensional black hole, i.e., the $c=1$ Liouville string in a flat two-dimensional target-space background, is known to be described by a completely integrable (and soluble) one-dimensional (quantum-mechanical) matrix model. The latter is essentially a theory of free fermionic fields $\psi(t)$ interacting with an inverted harmonic oscillator potential. The fermions depend on the Liouville dimension, which is a 'spatial' coordinate $r$ in this case. The $W_{\infty}$ charges in that case are described by the (infinite) set of the moments of the energy (Hamiltonian). In fact, if one considers a state with incoming fermions of energies $\varepsilon_{i}, i=1, \ldots k$, the conserved charges are

$$
Q_{n}=\sum_{i=1}^{k} \varepsilon_{i}^{n}
$$

which are conserved for each $n=1,2 \ldots \infty$, where the charge $n=1$ is the Hamiltonian of the system.

The $W_{\infty}$ symmetry algebra associated with these charges has a classical limit that is associated with the canonical transformations that preserve the free-fermion phase-space (area-preserving phase-space form), as we have discussed previously. From the detailed string theory considerations of the associated Liouville theory described previously, we know that there exist discrete gauge states that carry these charges $[39,40]$, i.e., the $Q_{n}(5.22)$ can couple to gauge fields, which constitute elements of the gauged $W_{1+\infty}$ Lie algebras discussed above, leading to the $w$ hair of the black hole [10]. In the context of the matrix model, the space-time dimension of the gauge field is $d=1$. The embedding of such matrix models in four dimensions can then be done by identifying the internal colour space $z, \bar{z}$ with the horizon surface of the four-dimensional black hole, whose asymptotic state is described by the $\mathrm{c}=1$ Liouville string theory.

\subsection{Hawking radiation from spherically-symmetric black holes and $W_{1+\infty}$ symmetries}

We close this Section by mentioning some important results that provide a link between phase-space-area-preserving $W_{1+\infty}$ algebras and Hawking radiation in generic four-dimensional 
black holes with spherically symmetric horizons, which aids understanding the connection between phase-space $W_{1+\infty}$ symmetries and area-preserving diffeomorphisms of the horizon in a more generic context than the $S U(\infty)$ black hole case discussed previously. It was shown in $[41,42]$ that moments of the Hawking radiation emitted by a generic, non-stringy spherically-symmetric black hole are connected to a $W_{1+\infty}$ algebra carried by higher-spin states, whose currents are sourced by background fields of higher-spin states, which can be identified with the discrete gauge states [39] discussed in our stringy approach [10] to black-hole singularities.

Crucial to the connection of Hawking radiation to $W_{\infty}$ algebras is the effective two-dimensional conformal field theory representation [43] of the dynamics of matter fields in the near-horizon geometry of a spherically-symmetric black hole, as adopted in our approach [10]. Within this context, it is known that the quanta of Hawking radiation emitted from the horizon of a sphericallysymmetric black hole break general covariance. As shown in [43], this symmetry is restored (in the sense that the corresponding gravitational anomalies in the quantum gravity path integral are cancelled) in the case of a $(1+1)$-dimensional black body at the black-hole Hawking temperature [2]. Thus, one can represent the effective two-dimensional field theory of the Hawking radiation on the black-hole horizon as a two-dimensional field theory with an infinity of two-dimensional conformal quantum fields with a thermal spectrum, with the left movers corresponding to radially infalling matter and right movers to outgoing matter.

Schwarzschild black holes emit Hawking radiation with a Planck distribution

$$
N^{ \pm}(\omega)=\frac{1}{e^{\beta \omega} \pm 1}
$$

where $\beta$ is the Hawking temperature [2], $\omega$ is the frequency (energy) of the radiation quantum, and $+(-)$ corresponds to fermions (bosons) respectively. The full spectrum of the (Fourier-analyzed) radiation is encoded in the higher moments or fluxes [41]. The energy flux, for instance, is given by the second moment of $N^{ \pm}(\omega), F_{2}(\omega)=\int_{0}^{\infty} \frac{d \omega}{2 \pi} \omega N^{ \pm}(\omega)$. The complete thermal Hawking radiation spectrum is specified by the infinity of higher moments:

$$
F_{2 n}^{+}=\int_{0}^{\infty} \frac{d \omega}{2 \pi} \omega^{n-1} N^{+}(\omega)=\left(1-2^{1-2 n}\right) \frac{B_{2 n}}{8 \pi n} \kappa^{2 n}
$$

or

$$
F_{2 n}^{-}=\int_{0}^{\infty} \frac{d \omega}{2 \pi} \omega^{n-1} N^{-}(\omega)=\frac{B_{2 n}}{8 \pi n} \kappa^{2 n}
$$

where the $B_{2 n}$ are the Bernoulli numbers and $\kappa=2 \pi / \beta$ is the surface gravity of the black hole.

An interesting proposal was made in [41] that the higher fluxes $F_{2 n}, n>1$ could be connected to phenomenological higher-spin currents (with appropriate normal ordering), i.e. higher-spin generalizations of the energy-momentum tensor, which is the spin-two current associated with the energy flux. As shown in [41], these higher currents can be expressed in terms of two dimensional boson and fermion fields, involving linear combinations of (two-dimensional) space-time derivatives acting on the fields. In an appropriate light-cone coordinate $(u, v)$ description, the outgoing near-horizon Hawking radiation is described by holomorphic ( $u$-dependent) currents of the following form in the scalar case [41]:

$$
J_{u u \ldots u}^{B}=\text { linear combinations of }:(-1)^{n+m} \partial_{u}^{m} \phi \partial_{u}^{2 n-m} \phi:,
$$


where $: \ldots$ : denotes appropriate normal ordering, as defined in $[41]^{9}$.

It should be noted that there are ambiguities in the representation of the higher moments of the Hawking radiation in terms of conformal fields on the horizon, reflected in the relative coefficients of the various terms appearing in the holomorphic currents (5.26). Moreover, the currents are not normalizable in general. However, these issues have been resolved by the proposal of [42] that the coefficients of these currents be fixed by a symmetry principle, namely by the postulate that there is a higher symmetry in the horizon of the black hole than the Virasoro symmetry, namely a $W_{\infty}$ algebra. It was to be expected from their higher-derivative holomorphic structure, $\partial_{z}^{n} \chi(\chi=$ $\phi, \psi)[24]$ that the currents could be cast in such a way as to form an infinite-dimensional algebra.

In a flat two-dimensional space-time, after Euclideanisation and replacing the light-cone coordinates $u, v$ by the complex variables $z, \bar{z}$ respectively, the $w_{\infty}$-generating bosonic currents for the conformal spin $s$ states can be written in the form

$$
j_{z \ldots z}^{(s) B}=q^{s-2} \frac{2^{s-3} s !}{(2 s-3) ! !} \sum_{k=1}^{s-1}(-1)^{k}\left[\frac{1}{s-1}(s)-1 k(s)-1 s-k\right]: \partial_{z}^{k} \phi(z) \partial_{z}^{s-k} \bar{\phi}(z)
$$

where :...: denotes normal ordering, defined in [42], and $q$ is a complex (in general) deformation parameter [24]. In this formalism the holomorphic free fields $\phi(z)$ are assumed to have two-point functions of the form $\left\langle\phi(z) \bar{\phi}\left(z^{\prime}\right)\right\rangle=-\ln \left(z-z^{\prime}\right)$, with the others vanishing. The deformation parameter $q$ can be fixed [42] by demanding that the currents (5.27), when covariantised as appropriate for the curved space-time of the spherical symmetric black hole (which is only conformally equivalent to a flat space), reproduce the higher moments of the Hawking flux. The $s=2$ current is independent of the $q$ deformation parameter, as expected from the fact that this current can be identified unambiguously with the holomoprphic stress tensor

$$
j_{u u}^{(2)}=-2 \pi T_{u u}^{\mathrm{hol}}
$$

However, the higher-spin currents depend on $q$, and one must fix $q=-i / 4$ [42] in order to reproduce the Hawking fluxes for bosons (5.25) when covariantising the expression (5.27) by replacing the ordinary derivatives with covariant ones, as is appropriate for discussing the Hawking flux in the conformally-flat metric that represents the near-horizon geometry of the black hole.

As discussed in great detail in [42], the currents (5.27) and their fermionic counterparts with spins higher than two are free of conformal or diffeomporphism anomalies (or, if the latter exist, they are trivial). This is consistent with the fact that the higher moments of the Hawking radiation are expected to describe a theory free of gravitational anomalies, since only the spin-two current (stress tensor) of the theory has diffeomorphism or conformal anomalies, and their cancellation required the appearance of the Hawking radiation spectrum [43]. Moreover, this is also consistent with the fact that, if these currents had conformal anomalies, they would correspond to new (nongauge) quantum numbers of the black hole, in violation of the no-hair theorem. Hawking radiation is consistent with this theorem, which is then reflected in the absence of anomalies in the currents corresponding to currents with spins higher than two.

\footnotetext{
${ }^{9}$ For the fermion case, see [41] and [44].
} 
The covariant higher-spin currents $J_{\mu_{1} \mu_{2} \ldots \mu_{n}}^{(s) B, F}$ are sourced by appropriate background fields $\mathscr{B}_{\mu_{1} \mu_{2} \ldots \mu_{n}}^{(s) B, F}$

$$
J_{\mu_{1} \ldots \mu_{n}}^{(s) B, F}=\frac{1}{\sqrt{g}} \frac{\delta}{\delta \mathscr{B}(s) B, F \mu_{1} \ldots \mu_{n}} S,
$$

where $S$ is the two-dimensional effective action of the Hawking radiation in the near-horizon geometry of the spherically-symmetric black hole. The relevant interactions in this effective geometry are then simply given by

$$
S_{\text {int }}=\int_{\text {near horizon 2D space-time }} d^{2} x \sqrt{g} \sum_{\alpha=B, F} \mathscr{B}^{(s) \alpha \mu_{1} \ldots \mu_{n}} J_{\mu_{1} \ldots \mu_{n}}^{(s) \alpha},
$$

and the background fields $\mathscr{B}^{(s)} \alpha \mu_{1} \ldots \mu_{n}$ may be taken taken to vanish at asymptotic spatial infinity. Eqn. (5.29) generalises the spin-2 case, in which the corresponding spin-2 current (the stress tensor) couples to the graviton field, $\int d^{2} x \sqrt{g} T^{\mu v} g_{\mu \nu}$, which is characterised by diffeomorphism invariance (acting as a 'gauge symmetry'): $\delta g_{\mu v}=\partial_{(\mu} \xi_{v)}$ for an infinitesimal diffeomorphism $\xi_{\mu} \rightarrow x_{\mu}+\xi_{\mu}$, provided the stress tensor is conserved ${ }^{10}$. As already remarked, the higher-spin currents are free from conformal and diffeomorphism anomalies [42] and are conserved exactly, and their conservation is associated with an infinity of Abelian gauge symmetries of the form

$$
\mathscr{B}_{\mu_{1} \ldots \mu_{n}}^{(s)} \rightarrow \mathscr{B}_{\mu_{1} \ldots \mu_{n}}^{(s)}+\partial_{\left(\mu_{1}\right.} \Xi_{\left.\mu_{2} \ldots \mu_{n}\right)}
$$

where the $(. .$.$) indicate the appropriate symmetrization of indices. The presence of these infinite$ gauge symmetries is consistent with the no-hair theorem, as the spatial integrals of the currents correspond to conserved charges. The existence of a $W_{\infty}$ symmetry of the matter in the nearhorizon geometry, which is larger than the Virasoro algebra, results in the complete integrability of the matter system, and is analogous to the case of matter in the near-horizon geometries of black-hole structures in the context of string theory [10,9], discussed above.

This $W_{\infty}$ algebra is phase-space-preserving, as are the $W_{\infty}$ algebras discussed in the stringy cases above. To see this, one may rewrite the (traceless) energy-momentum tensor of the twodimensional effective scalar theory using a point-splitting method [42]:

$$
\begin{aligned}
T_{\mu v} & =\lim _{y \rightarrow 0} \partial_{\mu} \phi(x-y) \partial_{v} \phi(x+y)-g_{\mu v}(\text { stress }- \text { tensor trace }) \\
& =\sum_{i=0} \sum_{j=0} \frac{(-1)^{i}}{i ! j !}: y^{\mu_{1}} \ldots y^{\mu_{i}} y^{v_{1}} \ldots y^{v_{j}} \partial_{\mu} \partial_{\mu_{1}} \ldots \partial_{\mu_{i}} \phi(x) \partial_{\nu} \partial_{v_{1}} \ldots \partial_{v_{j}} \phi(x):
\end{aligned}
$$

This expression is covariantised by replacing the partial derivatives by covariant derivatives, giving the right-hand-side of (5.31) a complicated expansion in terms of products of the higher-spin currents with $y$-dependent factors (5.31) that correspond via complicated background tensors $\mathscr{B}_{\mu_{1} \ldots \mu_{i n}}^{(s)}$ to the aforementioned background fields that source the higher-spin currents. For our purposes, the most important feature of (5.31) is the fact that the right-hand side depends not only on the coordinate $x^{\mu}$ but also on the quantity $y^{\mu}=d x^{\mu}$ in the cotangent bundle, and thus lives in a symplectic

\footnotetext{
${ }^{10}$ In the black-hole case, as we have discussed above, the diffeomorphism invariance is broken by the outgoing flux, but the form of the transformation is included in (5.30).
} 
phase-space manifold, showing that the $W_{1+\infty}$ algebra generated by the higher-spin currents of the Hawking radiation spectrum is a phase-space algebra.

One may associate these symmetries classically with horizon-area-preserving diffeomorphisms, following the discussion of Section 5.1 for the $\mathrm{SU}(\infty)$ coloured black hole case, but we reserve details for a future publication [44]. For our purposes here we only mention that the above-mentioned $W_{1+\infty}$ field theories on the black-hole horizon (for fermion or boson fields) may be "gauged", in the way discussed in [25] and reviewed in Section 5.3, by considering the extension of the fields in a space augmented with two extra dimensions $\xi, \bar{\xi}$ that can be taken to be the coordinates of the spherical horizon surface. The corresponding field theories of these $(d+2)$-dimensional fields, where $d$ the target-space dimensionality of the field theories on the horizon, are gauged in the way discussed in detail in [25] ${ }^{11}$ and can be shown to be invariant under the phase-space-areapreserving diffeomorphisms of the coordinates $\xi, \bar{\xi}$, which can be taken to represent the spherical black-hole horizon. It is in this way that the infinite-dimensional dynamical phase-space-areapreserving $W_{1+\infty}$ symmetries (5.31) of the near-horizon currents representing the Hawking radiation spectrum are related to classical horizon-preserving $w_{\infty}$ symmetry algebras. This is consistent with the identification of the classical black hole entropy (that is proportional to the area) with a classically-conserved Noether charge [27].

Before closing this discussion, we note an important difference of the two-dimensional $c=1$ string theory description $[4,6]$ of the black-hole singularity $[10,12]$ from a generic two-dimensional field-theoretic representation of the outgoing Hawking radiation, as discussed above. In the latter case, as we have seen, higher-spin currents consist of higher derivatives of propagating local (boson or fermion) fields, whereas in the $c=1$ string theory representation one encounters necessarily nonpropagating discrete delocalised states in the excitation spectrum, which are non-thermal. These states exist over and above the ordinary Hawking radiation fields and, as we have discussed above, their presence has highly non-trivial consequences. These delocalised states correspond to gauge states in the $c=1$ string theory [39], which carry the phase-space-area-conserving and coherencepreserving $W_{\infty}$ charges of the two-dimensional black hole singularity.

In our treatment, the singularity of the four-dimensional spherically-symmetric stringy black hole is characterised by an infinite-dimensional $W_{1+\infty}$ phase-space symmetry, whose charges are carried by the (infinity of) delocalised discrete, non-propagating higher-spin gauge states that are responsible for maintaining quantum coherence [10]. On the other hand, the black-hole horizon carries another set of phase-space $W_{1+\infty}$ symmetries, corresponding to higher-spin currents composed of the propagating modes of the two-dimensional effective field theory in the near-horizon geometry, representing the outgoing Hawking radiation flux. Thus, the Hawking radiation $W_{\infty}$ can be made to preserve the horizon area, but it is the discrete non-propagating string states that preserve quantum coherence.

\footnotetext{
${ }^{11}$ One has $d=1$ for the holomorphic fields that represent the outgoing Hawking radiation [43, 41, 42], which in the case of string-theory-inspired black holes [10] can be represented non-perturbatively via the $c=1$ matrix model [40].
} 


\section{Quo Vadis Supertranslations?}

\subsection{D-Brane Recoil and Supertranslations}

In order to understand better the microscopic mechanism for encoding information on the horizon, we consider the back-reaction of the black-hole horizon induced by its interaction with infalling matter. We first concentrate on the two-dimensional stringy black hole where the horizon is a point in space. As discussed in the previous section, we can represent the horizon of such a black hole as a D-particle defect in space, whereas the horizon of a four-dimensional black hole can be represented by a spherical Dirichlet brane. Using D-branes enables us to consider the 'momentary' capture of string matter by the horizon, in the sense of a spontaneous change of world-sheet boundary conditions from Neuman to Dirichlet. Such a process will lead to splitting of a closed-string state into two open ones. If one considers closed-string states as corresponding to gravitons and open-string states to gauge particles (including photons), this process may correspond to the conversion of an infalling graviton into a pair of photons. In general, when one represents the horizon of a black hole as a D-brane, the interaction with infalling string states implies a 'recoil' of the D-brane.

In the pilot case of a black hole in two target-space-time dimensions, we consider a matter particle represented by an open string falling into the horizon the interaction of the string with the D-particle horizon implies that at least one end of the open string attaches to the D-particle defect. As a result of the interaction, the D-particle undergoes a non-trivial change in velocity

$$
u_{r}=\frac{g_{s}}{M_{s}} \Delta p_{r}=\frac{g_{s}}{M_{s}} \xi_{r} p_{r},
$$

where $\xi_{r}$ denotes the fraction of the incident matter particle momentum that corresponds to the momentum transfer $\Delta p_{r}$ during the scattering. As discussed in [36] the non-trivial capture and splitting of the open string during its interaction with the D-particle, and the recoil of the latter, result in a local effective metric distortion of the form

$$
d s^{2}=g_{\mu \nu} d x^{\mu} d x^{v}=\left(\eta_{\mu \nu}+h_{\mu \nu}\right) d x^{\mu} d x^{v}: \quad h_{0 r}=u_{r} .
$$

In the black hole case of [4] with dilaton hair: $\Phi=-2 \ln \cosh r$, the string coupling $g_{s}=e^{\Phi}$ becomes weak at large distances: $g_{s} \rightarrow 0$ for $r \rightarrow \infty$. Hence $u_{i} \rightarrow 0$ and the space-time distortion vanishes at large distances, where the space is asymptotically flat.

The metric (6.2) can be generalised to higher dimensions, with a D-brane horizon recoiling along the $i$ 'th spatial dimension, in which case the space-time distortion due to the recoiling Dbrane horizon can be written as

$$
d s^{2}=d t^{2}+2 u_{i} d x^{i} d t-\delta_{i j} d x^{i} d x^{j}
$$

This metric was determined from world-sheet (logarithmic) conformal field theory considerations in $[36,35]$ : the world-sheet deformations representing the recoil of the D-brane close a logarithmic conformal algebra on the world-sheet of the string that represents a dragging of the reference frame by the D-brane horizon as it moves slowly on the flat space-time background. On the other hand, the string excitations represent relativistic particles, and thus they move at the local speed of light. 
One may perform a time coordinate change in the metric (6.3) to write it in the following form, which is valid up to terms $u^{3}$ for small recoil velocities $|\vec{u}| \ll 1$, as is appropriate for macroscopic black holes:

$$
d s^{2}=d t_{\mathrm{ff}}^{2}+2 u_{i} d x^{i} d t_{\mathrm{ff}}-\delta_{i j}\left(d x^{i}-u^{i} d t_{\mathrm{ff}}\right)\left(d x^{j}-u^{j} d t_{\mathrm{ff}}\right)+\mathscr{O}\left(u^{3}\right) .
$$

The metric (6.4) is the Gullstrand-Painlevé metric [45], which represents the geometry around the exterior of a Schwarzschild black hole. It represents the space falling into the black hole as a Gallilean 'river' on a flat space-time in which relativistic 'fish' may swim. The river represents the frame of the recoiling D-particle horizon, while the fish are the relativistic matter strings [46]. In (6.4), $t_{\mathrm{ff}}$ is the time of a free-floating observer who is at rest at infinity, compared to the centre of the black hole. We stress that, in the case of a black hole, the relative velocities $u^{i}$ are coordinatedependent, as already mentioned, due to the variation in the string coupling from being strong near the black hole singularity to being weak on the horizon.

In general, the recoil velocity has components normal and tangential to the horizon. The former can be associated with changes in the horizon area, and hence the black-hole entropy, whereas the latter would not change the area. In case of such a tangential recoil component, metrics of the form (6.4), written in Bondi coordinates, are of the same form as metrics that have been discussed in the past in connection with gravitational wave radiation in asymptotically-flat regions of space time $[16,17]$, and are known to be associated with supertranslations of the Bondi retarded time $u \equiv t_{\mathrm{ff}}+r$

$$
u \rightarrow u+\alpha(\theta, \phi),
$$

where $\alpha(\theta, \phi)$ is a function of the angular coordinates $\theta, \phi$. Such a retarded time was used in $[16,17]$ to discuss outgoing gravitational wave signals arriving at a distant observation point. Such BBMS $^{+}$transformations form an infinite-dimensional set of diffeomorphisms that include as a subgroup the four-parameter group of ordinary translations. In the case of matter falling into the black-hole horizon one may use instead the BBMS $^{-}$transformations pertaining to the advanced time $v=t_{\mathrm{ff}}-r$, which amount to the supertranslations

$$
v \rightarrow v+\xi(\theta, \phi),
$$

where $\xi(\theta, \phi)$ is a function of the angular coordinates $\theta, \phi$ on the black-hole horizon brane. The retarded (or advanced) time is viewed in general as a scalar function of the coordinates $u\left(x^{\mu}\right)$ that obeys $u_{, \mu} u_{, v} g^{\mu v}=0$, implying that the hypersurfaces $u=$ constant are light-like.

The generic space-times on which there are supertranslations that leave invariant the boundary conditions have the form [17]

$$
\begin{aligned}
d s^{2} & =\left(\frac{V}{r} e^{2 \beta}\right) d u^{2}-2 e^{2 \beta} d u d r+r^{2} h_{A B}\left(d x^{A}-U^{A} d u\right)\left(d x^{B}-U^{B} d u\right), \\
2 h_{A B} d x^{A} d x^{B} & =\left(e^{2 \beta}+e^{2 \gamma}\right) d \theta^{2}+4 \sin \theta \sinh (\gamma-\delta) d \theta d \phi+\sin ^{2} \theta\left(e^{-2 \beta}+e^{-2 \gamma}\right) d \phi^{2},
\end{aligned}
$$

where $u$ is a retarded time and $\left(r, x^{A}\right)$ are the three spatial coordinates, with $x^{A}=(\theta, \phi)$ the angular variables of the four-dimensional space-time appropriate for spherically-symmetric solutions of the gravitational equations, with determinant $\operatorname{det} h_{A B}=\sin ^{2} \theta$. The functions $V, U^{A}, \beta, \gamma, \delta$ are arbitrary functions of the coordinates and, to match them with (6.4), one performs the advanced (retarded) time transformation $u(v)$ from $t_{\mathrm{ff}}$ as mentioned previously. The asymptotic flatness of (6.4), due to 
the asymptotic vanishing of the recoil-induced distortion of the space-time surrounding the black hole horizon, implies the boundary conditions $\operatorname{Lim}\left(V / r=1, \operatorname{Lim}\left(r U^{A}\right)=\beta=\gamma=\delta\right)=0$ for fixed $u$ or $v$ and $r \rightarrow \infty$.

\subsection{Supertranslations are not Enough}

Hawking [18] has suggested that such supertranslations of an advanced Bondi time on the black-hole horizon may solve the issue of quantum coherence. The proposal builds upon analyses in the asymptotically-flat regime of a generic Schwarzschild black hole space-time by Strominger and collaborators $[14,15]$, who have provided arguments that the horizon supertranslation transformations can be viewed as a conformal Kac-Moody symmetry group, entailing an infinity of conservation laws. This symmetry group is an infinite-dimensional diffeomorphism group that leaves invariant the asymptotic BMS states as well as the generic quantum gravity scattering matrix defined by means of asymptotic in and out quantum states. Section 6 of the first reference in [15] made an association of these infinite supertranslation charges with hair for black holes, and suggested that such charges 'may bear on the information puzzle' ${ }^{12}$.

This suggestion looks similar in nature to the one we described above and in our previous works $[10,12]$, but there are important differences, and we do not think that supertranslations are sufficient to retain quantum coherence.

The supertranslations (6.5), (6.6) are symmetries of the asymptotic (large $r$ ) metrics and not of the full black hole background space-time, since they correspond to physically-inequivalent metrics, as discussed in $[16,17]$. This may be viewed as a sort of 'spontaneous breaking' of the supertranslation symmetries of the asymptotic Minkowski space-time by the black hole background. The associated Goldstone bosons have been identified tentatively (in the semiclassical black-hole limit of infinite entropy) [19] with delocalised graviton states of infinite wavelength. This proposal may sound similar to our topological stringy states, though the situation for a finite-area (finite-entropy) black hole is far from clear.

However, in our picture the discrete topological string states include such soft graviton states as a subset, along with an infinity of other higher-spin discrete (topological) states, all corresponding to conserved charges. These higher-spin states are separated by mass gaps $\propto n M_{s} / g_{s}, n \in Z^{+}$. Hence, given that on the horizon the string coupling is weak, such states may seem to be decoupled at the level of the local effective field theory level (LEFT), leaving only the massless graviton states as relevant. However, we conjecture that, for information retention at a finite-area black-hole horizon, one must consider the entirety of the delocalised (topological) higher-spin states, whose treatment goes beyond LEFT. These realize an infinite-dimensional area-preserving quantum $W_{\infty}$ symmetry algebra (with its infinity of conserved charges) in the coset black-hole model. This

\footnotetext{
${ }^{12}$ We know no obvious association of Kac-Moody algebras to the $W_{\infty}$ area-preserving algebras discussed here, except in the supersymmetric case considered in [47], where an area-preserving diffeomorphism algebra $\operatorname{SDiff}(\mathscr{M})$ of a twodimensional surface $\mathscr{M}$ acts as a derivation algebra on a super-Kac-Moody algebra, much as Virasoro algebras act as derivation algebras on Kac-Moody algebras on a one-dimensional circle $S^{1}$. The implications of this result in our case are not obvious. However, we recall that when one supersymmetrises the two-dimensional black hole to a (twisted topological) $N=2$ theory, a double $W_{1+\infty} \otimes W_{1+\infty}$ describes the singularity structure [48], which is broken $\rightarrow W_{1+\infty}$ away from it. The findings of [47] may be relevant in the case when this $N=2$ model is embedded in four dimensions. However, any connection between the supertranslation U(1) Kac-Moody algebra on the black-hole horizon [14] with (super-) $W_{\infty}$ horizon-area-preserving diffeomorphisms is currently unclear.
} 
picture is in agreement with the representation of the two-dimensional black hole horizon in four space-time dimensions as the world-sheet of a string, where a classical $w_{\infty}$ world-sheet symmetry preserves the area, and thus ensures information retention. Such world-sheet $w_{\infty}$ symmetries can be elevated to target space by means of appropriate world-sheet deformations, corresponding to the various excitations of the higher-spin target-space states [13]. Moreover, we have argued that a recoil displacement of a D-brane due to a 'sudden' impulse, as is the case of a string splitting on the horizon brane, merely mislays information that is stored on the horizon [37], in the sense that entanglement is induced between the recoiling quantum D-brane horizon and the quantum matter subsystem. The precise reproduction of the finite-area black-hole entropy for the generic four-dimensional black-hole case remains to be worked out (although arguments have been given in this direction for the two-dimensional stringy black hole in [12]), and we plan to return to this issue in the near future.

We consider that our view of discrete W-hair and the representation of the black-hole horizon as a $\mathrm{D}$ (irichlet) brane recoiling under the interaction with infalling matter is more appropriate than supertranslation invariance for maintaining quantum coherence. We would argue that any approach based on general relativity alone is limited in scope, and that string-theoretical considerations based on the rigorous counting of black-hole microstates, such as those outlined above, constitute a much more promising approach to the black-hole information-loss problem. In particular, we recall that the $W_{\infty}$ algebra corresponds to an $\mathrm{SU}(N \rightarrow \infty)$ algebra, as discussed in Section 4.4, and that the superstranslations (and the corresponding U(1) gauge states) do not include candidates for all the infinitely-coloured hair discussed there.

\section{Conclusions and Outlook}

We have reviewed and extended in this paper our previous arguments for the importance of infinite-dimensional $W_{\infty}$ symmetry in information retention by stringy black holes. This symmetry preserves two-dimensional area. As such, it plays a key rôle at the world-sheet level, 'balancing the information books' [10] in the two-dimensional black-hole model of [4], which may be elevated to four dimensions to describe a spherically-symmetric black hole [12]. In this case, the entropy is proportional to the area of the horizon, so entropy is therefore clearly conserved by a $W_{\infty}$ symmetry. The same symmetry preserves the area of the two-dimensional phase space describing a fermion interacting with a four-dimensional extremal black hole in the context of $N=2, D=4$ supergravity [9].

As we have stressed in this paper, there are still many open issues in our approach to the blackhole information problem based on $W_{\infty}$ symmetry, but we consider it to be much more complete and promising than recent suggestions $[14,15]$ based on supertranslations [16]. The $W_{\infty}$ symmetry is firmly embedded within string theory, which must surely be taken into account in any resolution of the black-hole information problem. Also, as can be seen from its relation to $\mathrm{SU}(\infty), W_{\infty}$ symmetry certainly offers conserved charges ( $W$-hair) that are not provided by supertranslations.

We have argued elsewhere that the $W$-hair of a four-dimensional black hole is in principle measurable [13], though this may not be feasible in practice [11]. For this reason, we consider that black holes 'mislay' information rather than 'lose' it. However, we still lack a specific 'Ariadne's 
thread' of external measurements that can enable us to reconstruct the information 'mislaid' within the black-hole labyrinth.

\section{Acknowledgement}

The authors thank George Leontaris for giving us the opportunity to meet and discuss these issues at Planck 2015, and to present them in these Proceedings.

\section{References}

[1] J. D. Bekenstein, Lett. Nuovo Cim. 4, 737 (1972); Phys. Rev. D 7, 2333 (1973); ibid. 9, 3292 (1974).

[2] S. W. Hawking, Commun. Math. Phys. 43, 199 (1975) [Commun. Math. Phys. 46, 206 (1976)]; Phys. Rev. D 13, 191 (1976); J. M. Bardeen, B. Carter and S. W. Hawking, Commun. Math. Phys. 31, 161 (1973);

[3] S. W. Hawking, Phys. Rev. D 14, 2460 (1976); S. W. Hawking, Commun. Math. Phys. 87, 395 (1982).

[4] E. Witten, Phys. Rev. D 44, 314 (1991) and article in Mansouri, F. (ed.), Scanio, J.J. (ed.): Quantum gravity and beyond 126-142 (1992) [hep-th/9206069].

[5] P. Horava, Phys. Lett. B 289, 293 (1992) [hep-th/9203031].

[6] S. Chaudhuri and J. D. Lykken, Nucl. Phys. B 396, 270 (1993) [hep-th/9206107].

[7] S. Kalara and D. V. Nanopoulos, Phys. Lett. B 267 (1991) 343.

[8] There are many important references in this field that we cannot cite here due to lack of space; some characteristic ones, of direct relevance to the material presented here, include: G. T. Horowitz and A. Strominger, Nucl. Phys. B 360, 197 (1991); A. Strominger and C. Vafa, Phys. Lett. B 379, 99 (1996) [hep-th/9601029]; C. G. Callan and J. M. Maldacena, Nucl. Phys. B 472, 591 (1996) [hep-th/9602043]; J. C. Breckenridge, D. A. Lowe, R. C. Myers, A. W. Peet, A. Strominger and C. Vafa, Phys. Lett. B 381, 423 (1996) [hep-th/9603078]; J. C. Breckenridge, R. C. Myers, A. W. Peet and C. Vafa, Phys. Lett. B 391, 93 (1997) [hep-th/9602065]; V. Balasubramanian and F. Larsen, Nucl. Phys. B 478, 199 (1996) [hep-th/9604189]; J. M. Maldacena and L. Susskind, Nucl. Phys. B 475, 679 (1996) [hep-th/9604042]; I. R. Klebanov and A. A. Tseytlin, Nucl. Phys. B 475, 179 (1996) [hep-th/9604166]; R. Emparan, G. T. Horowitz and R. C. Myers, Phys. Rev. Lett. 85, 499 (2000) [hep-th/0003118]; JHEP 0001, 007 (2000) [hep-th/9911043]; JHEP 0001, 021 (2000) [hep-th/9912135].

[9] S. Cacciatori, D. Klemm and D. Zanon, Class. Quant. Grav. 17, 1731 (2000) [hep-th/9910065].

[10] J. R. Ellis, N. E. Mavromatos and D. V. Nanopoulos Phys. Lett. B 267, 465 (1991).

[11] J. R. Ellis, N. E. Mavromatos and D. V. Nanopoulos Phys. Lett. B 293, 37 (1992) [hep-th/9207103]. hep-th/9403133 [Proc., International School of Subnuclear Physics: 31st Course: From Supersymmetry to the Origin of Space-Time 4-12 Jul 1993, Erice, Italy ( Edited by Antonio Zichichi. River Edge, N.J., World Scientific, 1995 (Subnuclear Series, v. 31)].

[12] J. R. Ellis, N. E. Mavromatos and D. V. Nanopoulos, Phys. Lett. B 278, 246 (1992) [hep-th/9112062].

[13] J. R. Ellis, N. E. Mavromatos and D. V. Nanopoulos, Phys. Lett. B 284, 27 (1992). Phys. Lett. B 284, 43 (1992) [hep-th/9203012]. 
[14] A. Strominger, JHEP 1407, 152 (2014) [arXiv:1312.2229 [hep-th]]. D. Kapec, V. Lysov, S. Pasterski and A. Strominger, JHEP 1408, 058 (2014) [arXiv:1406.3312 [hep-th]].

[15] A. Strominger and A. Zhiboedov, arXiv:1411.5745 [hep-th]; D. Kapec, V. Lysov, S. Pasterski and A. Strominger, arXiv:1502.07644 [gr-qc].

[16] H. Bondi, M. G. J. van der Burg and A. W. K. Metzner, Proc. Roy. Soc. Lond. A 269, 21 (1962).

[17] R. K. Sachs, Proc. Roy. Soc. Lond. A 270, 103 (1962).

[18] S. W. Hawking, arXiv:1509.01147 [hep-th].

[19] G. Dvali, C. Gomez and D. Luest, arXiv:1509.02114 [hep-th].

[20] G. t. Hooft, arXiv:1509.01695 [gr-qc].

[21] J. Polchinski, arXiv:1505.08108 [hep-th].

[22] F. Englert and P. Spindel, JHEP 1012, 065 (2010) [arXiv:1009.6190 [hep-th]].

[23] R. Brustein and A. J. M. Medved, JHEP 1506, 089 (2015) [arXiv:1503.05597 [hep-th]]; JHEP 1508, 082 (2015) [arXiv:1505.07131 [hep-th]].

[24] I. Bakas, Phys. Lett. B 228, 57 (1989); Commun. Math. Phys. 134, 487 (1990); C. N. Pope, L. J. Romans and X. Shen, A Brief history of W(infinity) Conf. Proc. C 9003122, 287 (1990); E. Bergshoeff, M. P. Blencowe and K. S. Stelle, Commun. Math. Phys. 128, 213 (1990); E. Sezgin, Lectures in Trieste 1991, Proceedings, High energy physics and cosmology, vol. 2 879-902 (1992) and Texas A \& M Univ. College Station - CTP-TAMU-92-013 (92/02) (204444) [hep-th/9202086]; I. Bakas and E. Kiritsis, Int. J. Mod. Phys. A 7S1A, 55 (1992) [Int. J. Mod. Phys. A 7, 55 (1992)] [hep-th/9109029].

[25] A. Kavalov and B. Sakita, Annals Phys. 255, 1 (1997) [hep-th/9603024] and references therein.

[26] I. R. Klebanov and A. M. Polyakov, Mod. Phys. Lett. A 6, 3273 (1991) [hep-th/9109032].

[27] R. M. Wald, Phys. Rev. D 48, 3427 (1993) [gr-qc/9307038].

[28] E. G. Floratos, J. Iliopoulos and G. Tiktopoulos, Phys. Lett. B 217, 285 (1989).

[29] J. Iliopoulos, Fortsch. Phys. 54, 139 (2006) [hep-th/0506005]; E. G. Floratos and J. Iliopoulos, Phys. Lett. B 632, 566 (2006) [hep-th/0509055].

[30] N. E. Mavromatos and E. Winstanley, Class. Quant. Grav. 17, 1595 (2000) [hep-th/9909018].

[31] B. Whitt, Phys. Rev. D10 (1988), 3000;

J. T. Wheeler, Nucl. Phys. B273 (1986), 732.

[32] C. W. Misner, K.S. Thorne and J.A. Wheeler, Gravitation (W.H. Freeman and Co., 1973).

[33] A.M. Polyakov, Mod. Phys. Lett. A6, 635 (1991).

[34] J. Schwinger, On Angular Momentum, US-AEC NYOC 3071 (1952).

[35] I. I. Kogan, N. E. Mavromatos and J. F. Wheater, Phys. Lett. B 387, 483 (1996) [hep-th/9606102].

[36] J. R. Ellis, N. E. Mavromatos and D. V. Nanopoulos, Gen. Rel. Grav. 32, 127 (2000) [gr-qc/9904068].

[37] J. R. Ellis, N. E. Mavromatos and D. V. Nanopoulos, Int. J. Mod. Phys. A 13, 1059 (1998) [hep-th/9609238].

[38] For a review see: O. Aharony, S. S. Gubser, J. M. Maldacena, H. Ooguri and Y. Oz, Phys. Rept. 323, 183 (2000) [hep-th/9905111], and references therein. 
[39] T. D. Chung and J. C. Lee, Phys. Lett. B 350, 22 (1995) [hep-th/9412095].

[40] S. R. Das, A. Dhar, G. Mandal and S. R. Wadia, Mod. Phys. Lett. A 7, 937 (1992) [Mod. Phys. Lett. A 7, 2245 (1992)] [hep-th/9112052].

[41] S. Iso, T. Morita and H. Umetsu, Phys. Rev. D 75, 124004 (2007) doi:10.1103/PhysRevD.75.124004 [hep-th/0701272]; Phys. Rev. D 76, 064015 (2007) doi:10.1103/PhysRevD.76.064015 [arXiv:0705.3494 [hep-th]]; Nucl. Phys. B 799, 60 (2008) doi:10.1016/j.nuclphysb.2008.02.014 [arXiv:0710.0453 [hep-th]].

[42] L. and M. Cvitan, JHEP 0805, 071 (2008) doi:10.1088/1126-6708/2008/05/071 [arXiv:0804.0198 [hep-th]]; L. , M. Cvitan, S. Pallua and I. Smolic, JHEP 0812, 021 (2008) doi:10.1088/1126-6708/2008/12/021 [arXiv:0808.2360 [hep-th]]; also in PoS ISFTG , 003 (2009); the approach has also been generalised to Kerr (axisymmetric) black holes in: L., M. Cvitan, S. Pallua and I. Smolic, Phys. Rev. D 80, 084034 (2009) doi:10.1103/PhysRevD.80.084034 [arXiv:0907.3722 [hep-th]].

[43] S. P. Robinson and F. Wilczek, Phys. Rev. Lett. 95, 011303 (2005) doi:10.1103/PhysRevLett.95.011303 [gr-qc/0502074].

[44] , J. Ellis, N.E. Mavromatos and D.V. Nanopoulos, in preparation.

[45] A. J. S. Hamilton and J. P. Lisle, Am. J. Phys. 76, 519 (2008) [gr-qc/0411060].

[46] N. E. Mavromatos and S. Sarkar, Eur. Phys. J. C 73, no. 3, 2359 (2013) [arXiv:1211.0968 [hep-ph]].

[47] E. Ragoucy and P. Sorba, Lett. Math. Phys. 21, 329 (1991).

[48] J. R. Ellis, N. E. Mavromatos and D. V. Nanopoulos, Phys. Lett. B 288, 23 (1992) [hep-th/9205107]. 